\title{
A SHARP BILINEAR ESTIMATE FOR THE BOURGAIN-TYPE SPACE WITH APPLICATION TO THE BENJAMIN EQUATION
}

\author{
WENGU CHEN AND JIE XIAO
}

\begin{abstract}
This note shows the existence of a sharp bilinear estimate for the Bourgain-type space and gives its application to the optimal local well/illposedness of the Cauchy problem for the Benjamin equation.
\end{abstract}

\section{INTRODUCTION}

In the process of understanding the new function spaces and their applications to some nonlinear evolution equations discovered by J. Bourgain in his 1993 paper [6], we obtain

Theorem 1.1. For $\alpha, 0 \neq \beta, \gamma, \xi, s, b \in \mathbb{R}$ let $p(\xi)=\beta \xi^{3}-\alpha \xi|\xi|+\gamma \xi$ and $X_{s, b, p}$ be the Bourgain type space - the completion of all $C_{0}^{\infty}\left(\mathbb{R}^{2}\right)$ functions $f$ in the norm given by

$$
\|f\|_{X_{s, b, p}}=\left(\int_{\mathbb{R}} \int_{\mathbb{R}}\left(1+|\xi|^{2}\right)^{s}\left(1+|\tau-p(\xi)|^{2}\right)^{b}|\widehat{f}(\xi, \tau)|^{2} d \xi d \tau\right)^{1 / 2} .
$$

Then:

(i) For $s>-3 / 4$ there exists $b \in(1 / 2,1)$ such that the bilinear inequality

$$
\left\|\partial_{x}(u v)\right\|_{X_{s,(b-1)+, p}} \leq c\|u\|_{X_{s, b, p}}\|v\|_{X_{s, b, p}}
$$

holds with a constant $c>0$ depending only on $s$ and $b$, where $a \pm$ means $a \pm \epsilon$ for a sufficiently small $\epsilon>0$.

(ii) For any $s \leq-3 / 4$ and any $b \in \mathbb{R}$ there is no constant $c>0$ depending only on $s$ and $b$ such that the bilinear inequality (1.1) holds.

Here $\widehat{f}(\xi, \tau)$ is the Fourier transform in $(\xi, \tau)$. And, it is worth pointing out that if $(\alpha, \beta, \gamma)=(0,1,0)$ then Theorem [1.1 (i) yields Kenig-Ponce-Vega's 17, Theorem 1.1], and Theorem 1.1 (ii) implies Kenig-Ponce-Vega's [17, Theorem 1.3] and Nakanishi-Takaoka-Tsutsumi's 21, Theorem 1 (i)]. Theorem 1.1 may seem rather specialized, but it is useful in connection with the well/ill-posedness of the Benjamin equation. More precisely, this theorem, together with other things, derives

Theorem 1.2. For $\alpha, \beta, \gamma, \xi, s, b \in \mathbb{R}$ with $\alpha \beta \neq 0$ let $p(\xi)=\beta \xi^{3}-\alpha \xi|\xi|+\gamma \xi$ and $H^{s}(\mathbb{R})$ be the square Sobolev space with order $s$ - the completion of all $C_{0}^{\infty}(\mathbb{R})$

2000 Mathematics Subject Classification. Primary 35Q53.

Key words and phrases. Sharp, bilinear estimate, Bourgain type space, local well/ill-posedness, Benjamin equation, $[k ; Z]$ multiplier.

The first/second-named author is supported in part by NNSF of China (No.10771130)/NESEC of Canada (No. RGPIN/261100-2003). The project is completed during the first-named author's visit to Memorial University of Newfoundland under the second-named author's research funding. 
functions $f$ under the norm

$$
\|f\|_{H^{s}}=\left(\int_{\mathbb{R}}\left(1+|\xi|^{2}\right)^{s}|\widehat{f}(\xi)|^{2} d \xi\right)^{\frac{1}{2}} .
$$

Then:

(i) For $s>-3 / 4$ and $u_{0} \in H^{s}(\mathbb{R})$ there exist $b \in(1 / 2,1)$ and $T=T\left(\left\|u_{0}\right\|_{H^{s}}\right)>0$ such that the Cauchy problem for the Benjamin equation:

$$
\left\{\begin{array}{l}
\partial_{t} u-\gamma \partial_{x} u+\alpha \mathcal{H} \partial_{x}^{2} u+\beta \partial_{x}^{3} u+\partial_{x}\left(u^{2}\right)=0, \quad(x, t) \in \mathbb{R} \times \mathbb{R}, \\
u(x, 0)=u_{0}(x), \quad x \in \mathbb{R},
\end{array}\right.
$$

has a unique solution $u$ in $C\left([0, T] ; H^{s}(\mathbb{R})\right) \cap X_{s, b, p}$.

(ii) For $s<-3 / 4$ the solution map of the above Cauchy problem is not continuous at zero, namely, there is no $T>0$ such that the solution map of (1.2):

$$
u_{0} \in H^{s}(\mathbb{R}) \mapsto u \in C\left([0, T] ; H^{s}(\mathbb{R})\right)
$$

is continuous at zero.

Here $\mathcal{H}$ denotes the one-dimensional Hilbert transform defined by

$$
\mathcal{H} f(x)=\lim _{\epsilon \rightarrow 0} \frac{1}{\pi} \int_{|y|>\epsilon} f(x-y) y^{-1} d y, \quad x \in \mathbb{R} .
$$

Furthermore, four things are worth noting. The first is that the above Benjamin equation is essentially the original Benjamin equation [5] which physically characterizes the vertical displacement (bounded above and below by rigid horizontal planes) of the interface between a thin layer of fluid atop and a much thicker layer of higher density fluid (cf. [22]) - see [1]-[3]-[3]-[9]-[20] for the study of existence, stability and asymptotics of solitary wave solutions of (1.2). The second is that setting $\alpha \neq 0$ and $\beta=0$ in (1.2) generates the Benjamin-Ono equation - see Kenig's survey [15] but also Ionescu-Kenig [14] and Burq-Planchon [8] for most recent developments. The third is that: if $(\beta, \gamma)=(-1,0)$ and $\alpha=-\nu \in(-1,0)$ then Theorem 1.2 (i) goes back to Kozono-Ogawa-Tanisaka's [18, Theorem 2.1]; if $s \geq-1 / 8$ and $\gamma=0$ then Theorem 1.2 (i) returns to Guo-Huo's [13, Theorem 1.1]; if $(s, \gamma)=(0,0)$ and $\alpha \beta>0$ then Theorem 1.2 (i) is the local part of the well-posedness in Linares [19. The fourth is that Theorem 1.2 is sharp in the sense that $s>-3 / 4$ and $s<-3 / 4$ deduce the positive and negative aspects of the posedness of (1.2) respectively Theorem 1.2 (ii) is a new discovery and achieved via Bejenaru-Tao's argument for [4. Theorem 2] plus an example in Bourgain [7] and Tzvetkov [25] - in the near future we will handle the intermediate index $s=-3 / 4$ although it is our conjecture that Theorem 1.2 (i) can extend to this value at least in the distributional sense just like one in Christ-Colliander-Tao's paper 10 on the Korteweg-de Vries (KdV) equation which is recovered from taking $(\alpha, \beta, \gamma)=(0,1,0)$ in (1.2).

In order to prove Theorems 1.1 and 1.2 we apply $\mathrm{T}$. Tao's $[k ; Z]$-multiplier norm method (introduced in 23 to settle some problems for the typical dispersive equations including $\mathrm{KdV}$ ) - see the second and third sections of this paper, but also the linear estimates established in [16-12]-11] and the classical fixed point theorem - see the final section of this paper. For the sake of convenience, we will use the abbreviation $\langle\xi\rangle=\sqrt{1+|\xi|^{2}}$ for $\xi \in \mathbb{R}$, but also denote by $A \lesssim B$ the statement that $A \leq C B$ holds for some large constant $C$ which may vary from line to line and depend on various parameters; similarly use $A \ll B$ to represent $A \leq C^{-1} B$; and use $A \sim B$ to stand for $A \lesssim B \lesssim A$. Last but not least, we would 
like to acknowledge a couple of discussions with Q.-Y. Xue from Beijing Normal University.

\section{Fundamental Estimate for Dyadic Blocks}

From now on, for $Z$, an abelian additive group with an invariant measure $d \xi$, and for an integer $k \geq 2$, we denote by $\Gamma_{k}(Z)$ the hyperplane

$$
\Gamma_{k}(Z):=\left\{\xi=\left(\xi_{1}, \cdots, \xi_{k}\right) \in Z^{k}: \xi_{1}+\cdots+\xi_{k}=0\right\}
$$

which is equipped with the measure

$$
\int_{\Gamma_{k}(Z)} f(\xi):=\int_{Z^{k-1}} f\left(\xi_{1}, \cdots, \xi_{k-1},-\xi_{1}-\cdots-\xi_{k-1}\right) d \xi_{1} \cdots d \xi_{k-1}
$$

Following Tao 23 we say that a function $m: \Gamma_{k}(Z) \mapsto \mathbb{C}$ is just a $[k ; Z]-$ multiplier, and the multiplier norm $\|m\|_{[k ; Z]}$ is defined to be the minimal constant $\kappa \geq 0$ such that the inequality

$$
\left|\int_{\Gamma_{k}(Z)} m(\xi) \prod_{j=1}^{k} f_{j}\left(\xi_{j}\right)\right| \leq \kappa \prod_{j=1}^{k}\left\|f_{j}\right\|_{L^{2}(Z)},
$$

holds for all test functions $f_{j}$ on $Z$. Meanwhile, we need to review some of Tao's notations. Any summations over capitalized variables such as $N_{j}, L_{j}, H$ are presumed to be dyadic - that is to say - these variables range over numbers of the form $2^{k}$ for $k \in \mathbb{Z}$ (the set of all integers). If $N_{1}, N_{2}, N_{3}>0$ then $N_{\text {max }}, N_{\text {med }}, N_{\text {min }}$ stand for the maximum, median, and minimum of $N_{1}, N_{2}, N_{3}$ respectively, and hence $N_{\max } \geq N_{\text {med }} \geq N_{\min }$. Likewise, we have $L_{\max } \geq L_{\text {med }} \geq L_{\min }$ whenever $L_{1}, L_{2}, L_{3}>0$. More than that, we adopt the summation conventions as follows. Any summation of the form $L_{\max } \sim \cdots$ is a sum over the three dyadic variables $L_{1}, L_{2}, L_{3} \gtrsim 1$ : for example,

$$
\sum_{L_{\max } \sim H}:=\sum_{L_{1}, L_{2}, L_{3} \gtrsim 1: L_{\max } \sim H} .
$$

Similarly, any summation of the form $N_{\max } \sim \cdots$ sum over the three dyadic variables $N_{1}, N_{2}, N_{3}>0$ - in particular -

$$
\sum_{N_{\text {max }} \sim N_{\text {med }} \sim N}:=\sum_{N_{1}, N_{2}, N_{3}>0: N_{\text {max }} \sim N_{\text {med }} \sim N} .
$$

Finally, if $\tau, \xi$ and $p(\xi)$ are given with $\tau_{1}+\tau_{2}+\tau_{3}=0$, then we write

$$
\lambda:=\tau-p(\xi) \text { and } \quad \lambda_{j}:=\tau_{j}-p\left(\xi_{j}\right) \quad \text { for } \quad j=1,2,3 .
$$

In the sequel, we will establish the $[3 ; Z]$-multiplier norm estimate for the Benjamin equation. During estimation we need the resonance function

$$
h(\xi):=p\left(\xi_{1}\right)+p\left(\xi_{2}\right)+p\left(\xi_{3}\right)=-\lambda_{1}-\lambda_{2}-\lambda_{3},
$$

which arises from what extent the spatial frequencies $\xi_{1}, \xi_{2}, \xi_{3}$ share with one another. By the dyadic decomposition of each variable $\xi_{j}$ or $\lambda_{j}$, as well as the function $h(\xi)$, we are led to consider

$$
\|\mathcal{X}\|_{\left[3, \mathbb{R}^{2}\right]}:=\left\|X_{N_{1}, N_{2}, N_{3} ; H ; L_{1}, L_{2}, L_{3}}\right\|_{[3, \mathbb{R} \times \mathbb{R}]},
$$


where $X_{N_{1}, N_{2}, N_{3} ; H ; L_{1}, L_{2}, L_{3}}$ is the multiplier determined via

$$
X_{N_{1}, N_{2}, N_{3} ; H ; L_{1}, L_{2}, L_{3}}(\xi, \lambda):=\chi_{|h(\xi)| \sim H} \prod_{j=1}^{3} \chi_{\left|\xi_{j}\right| \sim N_{j}} \chi_{\left|\lambda_{j}\right| \sim L_{j}} .
$$

From the identities

$$
\xi_{1}+\xi_{2}+\xi_{3}=0 \quad \text { and } \quad \lambda_{1}+\lambda_{2}+\lambda_{3}+h(\xi)=0
$$

we see that $X_{N_{1}, N_{2}, N_{3} ; H ; L_{1}, L_{2}, L_{3}}$ vanishes unless

$$
N_{\text {max }} \sim N_{\text {med }} \text { and } L_{\max } \sim \max \left\{H, L_{\text {med }}\right\} .
$$

Consequently, from (2.1) we obtain the following algebraic smoothing relation.

Lemma 2.1. Let $\alpha, \beta, \gamma$ and $p(\xi)$ be the same as in Theorem 1.1. If $N_{\max } \sim$ $N_{\text {med }} \gtrsim \max \left\{1, \frac{4|\alpha|}{3|\beta|}\right\}$, then

$$
\max \left\{\left|\lambda_{1}\right|,\left|\lambda_{2}\right|,\left|\lambda_{3}\right|\right\} \gtrsim N_{1} N_{2} N_{3} .
$$

Proof of Lemma 2.1. Noticing

$$
p\left(\xi_{j}\right)=\beta \xi_{j}^{3}-\alpha \xi_{j}\left|\xi_{j}\right|+\gamma \xi_{j} \quad \text { for } \quad j=1,2,3,
$$

we have

$$
\begin{aligned}
h(\xi) & =-\lambda_{1}-\lambda_{2}-\lambda_{3} \\
& =p\left(\xi_{1}\right)+p\left(\xi_{2}\right)+p\left(\xi_{3}\right) \\
& =3 \beta \xi_{1} \xi_{2} \xi_{3}-\alpha\left(\xi_{1}\left|\xi_{1}\right|+\xi_{2}\left|\xi_{2}\right|+\xi_{3}\left|\xi_{3}\right|\right) .
\end{aligned}
$$

Next, we simplify the last formula according to six $\left(\xi_{1}, \xi_{2}\right)$-angle regions of $\mathbb{R}^{2}$ formed by three lines $\xi_{1}=0 ; \xi_{2}=0 ; \xi_{1}+\xi_{2}=0$.

$\angle 1$ : If $\xi_{1} \geq 0, \xi_{2} \geq 0$, then $N_{3}=N_{\max }$ and hence

$$
h(\xi)=3 \beta \xi_{1} \xi_{2} \xi_{3}-\alpha\left(\xi_{1}^{2}+\xi_{2}^{2}-\left(\xi_{1}+\xi_{2}\right)^{2}\right)=3 \beta \xi_{1} \xi_{2} \xi_{3}+2 \alpha \xi_{1} \xi_{2}=3 \beta \xi_{1} \xi_{2}\left(\xi_{3}+\frac{2 \alpha}{3 \beta}\right) .
$$

$\angle 2$ : If $\xi_{1} \leq 0, \xi_{2} \leq 0$, then $N_{3}=N_{\max }$ and hence

$$
h(\xi)=3 \beta \xi_{1} \xi_{2} \xi_{3}-\alpha\left(-\xi_{1}^{2}-\xi_{2}^{2}+\left(\xi_{1}+\xi_{2}\right)^{2}\right)=3 \beta \xi_{1} \xi_{2} \xi_{3}-2 \alpha \xi_{1} \xi_{2}=3 \beta \xi_{1} \xi_{2}\left(\xi_{3}-\frac{2 \alpha}{3 \beta}\right) .
$$

$\angle 3$ : If $\xi_{1} \geq 0, \xi_{2} \leq 0, \xi_{1}+\xi_{2} \geq 0$, then $N_{1}=N_{\max }$ and hence

$$
h(\xi)=3 \beta \xi_{1} \xi_{2} \xi_{3}-\alpha\left(\xi_{1}^{2}-\xi_{2}^{2}-\left(\xi_{1}+\xi_{2}\right)^{2}\right)=3 \beta \xi_{1} \xi_{2} \xi_{3}-2 \alpha \xi_{2} \xi_{3}=3 \beta \xi_{2} \xi_{3}\left(\xi_{1}-\frac{2 \alpha}{3 \beta}\right) .
$$

$\angle 4:$ If $\xi_{1} \geq 0, \xi_{2} \leq 0, \xi_{1}+\xi_{2} \leq 0$, then $N_{2}=N_{\max }$ and hence

$$
h(\xi)=3 \beta \xi_{1} \xi_{2} \xi_{3}-\alpha\left(\xi_{1}^{2}-\xi_{2}^{2}+\left(\xi_{1}+\xi_{2}\right)^{2}\right)=3 \beta \xi_{1} \xi_{2} \xi_{3}+2 \alpha \xi_{1} \xi_{3}=3 \beta \xi_{1} \xi_{3}\left(\xi_{2}+\frac{2 \alpha}{3 \beta}\right) .
$$

$\angle 5$ : If $\xi_{1} \leq 0, \xi_{2} \geq 0, \xi_{1}+\xi_{2} \geq 0$, then $N_{2}=N_{\max }$ and hence

$$
h(\xi)=3 \beta \xi_{1} \xi_{2} \xi_{3}-\alpha\left(-\xi_{1}^{2}+\xi_{2}^{2}-\left(\xi_{1}+\xi_{2}\right)^{2}\right)=3 \beta \xi_{1} \xi_{2} \xi_{3}-2 \alpha \xi_{1} \xi_{3}=3 \beta \xi_{2} \xi_{3}\left(\xi_{2}-\frac{2 \alpha}{3 \beta}\right) .
$$

$\angle 6$ : If $\xi_{1} \leq 0, \xi_{2} \geq 0, \xi_{1}+\xi_{2} \leq 0$, then $N_{1}=N_{\max }$ and hence

$$
h(\xi)=3 \beta \xi_{1} \xi_{2} \xi_{3}-\alpha\left(-\xi_{1}^{2}+\xi_{2}^{2}+\left(\xi_{1}+\xi_{2}\right)^{2}\right)=3 \beta \xi_{1} \xi_{2} \xi_{3}+2 \alpha \xi_{2} \xi_{3}=3 \beta \xi_{2} \xi_{3}\left(\xi_{1}+\frac{2 \alpha}{3 \beta}\right) .
$$


As a result, we find that

$$
N_{\text {max }} \sim N_{\text {med }} \gtrsim \max \left\{1, \frac{4|\alpha|}{3|\beta|}\right\}
$$

implies

$$
\max \left\{\left|\lambda_{1}\right|,\left|\lambda_{2}\right|,\left|\lambda_{3}\right|\right\} \geq \frac{1}{3}\left(\left|\lambda_{1}+\lambda_{2}+\lambda_{3}\right|\right) \gtrsim N_{1} N_{2} N_{3}
$$

whence getting (2.4).

Interestingly, Lemma 2.1 and its argument may allow us to assume that

$$
H \sim N_{\max }^{2} N_{\min }
$$

since the multiplier in (2.2) vanishes otherwise.

Now we are in the position to state the fundamental estimate on dyadic blocks.

Lemma 2.2. Let $\alpha, \beta, \gamma$ and $p(\xi)$ be the same as in Theorem 1.1. Suppose that

$$
\min \left\{H, N_{1}, N_{2}, N_{3}, L_{1}, L_{2}, L_{3}\right\}>0
$$

obey (2.3) and (2.5). Then:

(i) $\left((++)\right.$ Coherence) $N_{\max } \sim N_{\min } \& L_{\max } \sim H$ implies

$$
\|\mathcal{X}\|_{\left[3, \mathbb{R}^{2}\right]} \lesssim L_{\text {min }}^{1 / 2} N_{\text {max }}^{-1 / 4} L_{\text {med }}^{1 / 4} .
$$

(ii) ((+-) Coherence) Anyone of the following three conditions

$$
\left\{\begin{array}{l}
N_{1} \sim N_{2} \gg N_{3} \& H \sim L_{3} \gtrsim L_{2}, L_{1} ; \\
N_{2} \sim N_{3} \gg N_{1} \& H \sim L_{1} \gtrsim L_{2}, L_{3} ; \\
N_{3} \sim N_{1} \gg N_{2} \& H \sim L_{2} \gtrsim L_{3}, L_{1},
\end{array}\right.
$$

implies

$$
\|\mathcal{X}\|_{\left[3, \mathbb{R}^{2}\right]} \lesssim L_{\min }^{1 / 2} N_{\max }^{-1}\left(\min \left\{H, \frac{N_{\max }}{N_{\min }} L_{\text {med }}\right\}\right)^{1 / 2} .
$$

(iii) In all other cases, one has

$$
\|\mathcal{X}\|_{\left[3, \mathbb{R}^{2}\right]} \lesssim L_{\min }^{1 / 2} N_{\text {max }}^{-1}\left(\min \left\{H, L_{\text {med }}\right\}\right)^{1 / 2} .
$$

Proof of Lemma 2.2. In the high modulation case: $L_{\max } \sim L_{m e d} \gg H$ we have by the elementary estimate in [23, (37), p.861],

$$
\|\mathcal{X}\|_{\left[3, \mathbb{R}^{2}\right]} \lesssim L_{\text {min }}^{1 / 2} N_{\text {min }}^{1 / 2} \lesssim L_{\text {min }}^{1 / 2} N_{\text {max }}^{-1} N_{\text {min }}^{1 / 2} N_{\text {max }} \lesssim L_{\text {min }}^{1 / 2} N_{\text {max }}^{-1} H^{1 / 2}
$$

For the low modulation case: $L_{\max } \sim H$, by symmetry we may assume $L_{1} \geq$ $L_{2} \geq L_{3}$. By [23, Corollary 4.2], we have

$$
\begin{aligned}
\|\mathcal{X}\|_{\left[3, \mathbb{R}^{2}\right]} \lesssim & L_{3}^{1 / 2} \mid\left\{\xi_{2}:\left|\xi_{2}-\xi_{2}^{0}\right| \ll N_{\min } ;\left|\xi-\xi_{2}-\xi_{3}^{0}\right| \ll N_{\min }\right. \\
& \left.p\left(\xi_{2}\right)+p\left(\xi-\xi_{2}\right)=\tau+O\left(L_{2}\right)\right\}\left.\right|^{1 / 2}
\end{aligned}
$$

for some $\tau \in \mathbb{R}, \xi, \xi_{1}^{0}, \xi_{2}^{0}, \xi_{3}^{0}$ satisfying

$$
\left|\xi_{j}^{0}\right| \sim N_{j} \text { for } j=1,2,3 ;\left|\xi_{1}^{0}+\xi_{2}^{0}+\xi_{3}^{0}\right| \ll N_{\text {min }} ;\left|\xi+\xi_{1}^{0}\right| \ll N_{\text {min }} .
$$


To estimate the right-hand side of (2.9) we will employ the identity

$$
\begin{aligned}
p\left(\xi_{2}\right)+p\left(\xi-\xi_{2}\right) & =\left(\beta \xi_{2}^{3}-\alpha \xi_{2}\left|\xi_{2}\right|\right)+\left(\beta\left(\xi-\xi_{2}\right)^{3}-\alpha\left(\xi-\xi_{2}\right)\left|\xi-\xi_{2}\right|\right) \\
& =\beta\left(\xi^{3}-3 \xi^{2} \xi_{2}+3 \xi \xi_{2}^{2}\right)-\alpha\left(\xi_{2}\left|\xi_{2}\right|+\left(\xi-\xi_{2}\right)\left|\xi-\xi_{2}\right|\right) \\
& =q\left(\xi, \xi_{2}\right) .
\end{aligned}
$$

Now, an application of (2.9) and (2.10) yields

$$
q\left(\xi, \xi_{2}\right)=\tau+O\left(L_{2}\right) .
$$

Moreover, $q\left(\xi, \xi_{2}\right)$ can be calculated on four angle regions of $\mathbb{R}^{2}$ as follows.

$\angle 7$ : If $\xi_{2} \geq 0, \xi-\xi_{2} \geq 0$, then

$$
\begin{aligned}
q\left(\xi, \xi_{2}\right) & =\beta\left(\xi^{3}-3 \xi^{2} \xi_{2}+3 \xi \xi_{2}^{2}\right)-\alpha\left(\xi_{2}^{2}+\left(\xi-\xi_{2}\right)^{2}\right) \\
& =\beta\left(\xi^{3}-3 \xi^{2} \xi_{2}+3 \xi \xi_{2}^{2}\right)-\alpha\left(2 \xi_{2}^{2}-2 \xi \xi_{2}+\xi^{2}\right) \\
& =(3 \beta \xi-2 \alpha) \xi_{2}^{2}-(3 \beta \xi-2 \alpha) \xi \xi_{2}+\left(\beta \xi^{3}-\alpha \xi^{2}\right) \\
& =(3 \beta \xi-2 \alpha)\left(\xi_{2}-\frac{\xi}{2}\right)^{2}+\frac{1}{4}(\beta \xi-2 \alpha) \xi^{2} .
\end{aligned}
$$

$\angle 8:$ If $\xi_{2} \leq 0, \xi-\xi_{2} \leq 0$, then

$$
\begin{aligned}
q\left(\xi, \xi_{2}\right) & =\beta\left(\xi^{3}-3 \xi^{2} \xi_{2}+3 \xi \xi_{2}^{2}\right)+\alpha\left(\xi_{2}^{2}+\left(\xi-\xi_{2}\right)^{2}\right) \\
& =(3 \beta \xi+2 \alpha)\left(\xi_{2}-\frac{\xi}{2}\right)^{2}+\frac{1}{4}(\beta \xi+2 \alpha) \xi^{2} .
\end{aligned}
$$

$\angle 9:$ If $\xi_{2} \geq 0, \xi-\xi_{2} \leq 0$, then

$$
\begin{aligned}
q\left(\xi, \xi_{2}\right) & =\beta\left(\xi^{3}-3 \xi^{2} \xi_{2}+3 \xi \xi_{2}^{2}\right)-\alpha\left(\xi_{2}^{2}-\left(\xi-\xi_{2}\right)^{2}\right) \\
& =\beta\left(\xi^{3}-3 \xi^{2} \xi_{2}+3 \xi \xi_{2}^{2}\right)+\alpha\left(\xi^{2}-2 \xi \xi_{2}\right) \\
& =3 \beta \xi\left(\xi_{2}^{2}-\left(\xi+\frac{2 \alpha}{3 \beta}\right) \xi_{2}\right)+\left(\beta \xi^{3}+\alpha \xi^{2}\right) \\
& =3 \beta \xi\left(\xi_{2}-2^{-1}\left(\xi+\frac{2 \alpha}{3 \beta}\right)\right)^{2}+\left(\frac{\beta}{4} \xi^{3}-\frac{\alpha^{2}}{3 \beta} \xi\right) .
\end{aligned}
$$

$\angle 10$ : If $\xi_{2} \leq 0, \xi-\xi_{2} \geq 0$, then

$$
\begin{aligned}
q\left(\xi, \xi_{2}\right) & =\beta\left(\xi^{3}-3 \xi^{2} \xi_{2}+3 \xi \xi_{2}^{2}\right)-\alpha\left(-\xi_{2}^{2}+\left(\xi-\xi_{2}\right)^{2}\right) \\
& =3 \beta \xi\left(\xi_{2}-2^{-1}\left(\xi-\frac{2 \alpha}{3 \beta}\right)\right)^{2}+\left(\frac{\beta}{4} \xi^{3}-\frac{\alpha^{2}}{3 \beta} \xi\right) .
\end{aligned}
$$

With the help of these computations, we can reach (i)-(ii)-(iii) in Lemma 2.2 . First, if $N_{1} \sim N_{2} \sim N_{3}$, then (2.11) ensures that $\xi_{2}$ belongs to one interval of length $O\left(L_{2}^{1 / 2} N_{\max }^{-1 / 2}\right)$ no matter which one of the foregoing four cases holds, and hence

so (2.6) follows.

$$
\|\mathcal{X}\|_{\left[3, \mathbb{R}^{2}\right]} \lesssim L_{3}^{1 / 2} L_{2}^{1 / 4} N_{\text {max }}^{-1 / 4}=L_{\text {min }}^{1 / 2} L_{\text {med }}^{1 / 4} N_{\text {max }}^{-1 / 4},
$$

Next, if $N_{2} \sim N_{3} \gg N_{1}$, then

$$
\left|\xi_{2}-2^{-1}\left(\xi \pm \frac{2 \alpha}{3 \beta}\right)\right| \sim N_{2} .
$$


From (2.11) it follows that $\xi_{2}$ is in one interval of length $O\left(L_{2} N_{2}^{-1} N_{1}^{-1}\right)$, and hence

$$
\|\mathcal{X}\|_{\left[3, \mathbb{R}^{2}\right]} \lesssim L_{3}^{1 / 2} L_{2}^{1 / 2} N_{1}^{-1 / 2} N_{2}^{-1 / 2}=L_{\text {min }}^{1 / 2} L_{\text {med }}^{1 / 2} N_{\text {min }}^{-1 / 2} N_{\text {max }}^{-1 / 2} .
$$

But $\xi_{2}$ is also in an interval of length $\ll N_{\min }$. Therefore (2.7) follows.

Last, if $N_{1} \sim N_{2} \gg N_{3}$ or $N_{1} \sim N_{3} \gg N_{2}$, then

$$
\left|\xi_{2}-2^{-1} \xi\right| \sim N_{1},\left|\xi_{2}-2^{-1}\left(\xi \pm \frac{2 \alpha}{3 \beta}\right)\right| \sim N_{1},
$$

and hence $\xi_{2}$ is in one interval of length $O\left(L_{2} N_{\max }^{-2}\right)$ no matter which one of the foregoing four cases holds. This gives

$$
\|\mathcal{X}\|_{\left[3, \mathbb{R}^{2}\right]} \lesssim L_{\text {min }}^{1 / 2} L_{\text {med }}^{1 / 2} N_{\text {max }}^{-1},
$$

and consequently, (2.8) follows.

\section{Sharp Bilinear Estimate}

This section is devoted to verifying Theorem 1.1 .

Proof of Theorem 1.1 (i). First of all, Plancherel's formula tells us that proving (1.1) amounts to showing

$$
\left\|\frac{\left(\xi_{1}+\xi_{2}\right)<\xi_{1}>^{-s}<\xi_{2}>^{-s}<\xi_{3}>^{s}}{<\tau_{1}-p\left(\xi_{1}\right)>^{b}<\tau_{2}-p\left(\xi_{2}\right)>^{b}<\tau_{3}-p\left(\xi_{3}\right)>^{(1-b)-}}\right\|_{[3, \mathbb{R} \times \mathbb{R}]} \lesssim 1 .
$$

But, by the definition of $h(\xi)$ and the dyadic decomposition of each variable $\xi_{j}, \lambda_{j}$ where $j=1,2,3$, we may assume

$$
\left|\xi_{j}\right| \sim N_{j} ;\left|\lambda_{j}\right| \sim L_{j} ;|h(\xi)| \sim H
$$

So, using the translation invariance of the $[3 ; Z]$-multiplier norm, we can always restrict our estimate on

$$
\min \left\{L_{1}, L_{2}, L_{3}\right\} \gtrsim 1 \text { and } \max \left\{N_{1}, N_{2}, N_{3}\right\} \gtrsim \max \left\{1, \frac{4|\alpha|}{3|\beta|}\right\} .
$$

Now, the comparison principle and orthogonality from [23, Schur's test, p. 851] reduce proving the multiplier norm estimate (3.1) to showing that

$$
\sum_{N_{\text {max }} \sim N_{\text {med }} \sim N} \sum_{L_{\text {max }} \sim L_{\text {med }}} \sum_{H \ll L_{\text {max }}} \frac{N_{3}<N_{3}>^{s}\|\mathcal{X}\|_{\left[3 ; \mathbb{R}^{2} ; H\right]}}{<N_{1}>^{s}<N_{2}>^{s} L_{1}^{b} L_{2}^{b} L_{3}^{(1-b)-}} \lesssim 1
$$

and

$$
\sum_{N_{\text {max }} \sim N_{\text {med }} \sim N} \sum_{L_{1}, L_{2}, L_{3} \gtrsim 1} \frac{N_{3}<N_{3}>^{s}\|\mathcal{X}\|_{\left[3 ; \mathbb{R}^{2} ; L_{\max }\right]}}{<N_{1}>^{s}<N_{2}>^{s} L_{1}^{b} L_{2}^{b} L_{3}^{(1-b)-}} \lesssim 1
$$

hold for all $N \gtrsim \max \left\{1, \frac{4|\alpha|}{3|\beta|}\right\}$, where

$$
\|\mathcal{X}\|_{\left[3 ; \mathbb{R}^{2} ; H\right]}:=\left\|X_{N_{1}, N_{2}, N_{3} ; H ; L_{1}, L_{2}, L_{3}}\right\|_{[3 ; \mathbb{R} \times \mathbb{R}]}
$$

and

$$
\|\mathcal{X}\|_{\left[3 ; \mathbb{R}^{2} ; L_{\max }\right]}:=\left\|X_{N_{1}, N_{2}, N_{3} ; L_{\max } ; L_{1}, L_{2}, L_{3}}\right\|_{[3 ; \mathbb{R} \times \mathbb{R}]} .
$$

Next, we verify (3.3) and (3.2). In fact, this can be accomplished by Lemma 2.2 and the following delicate summations in which we always fix $N \gtrsim \max \left\{1, \frac{4|\alpha|}{3|\beta|}\right\}$ and consequently have (2.5). 
We first prove (3.2). By (2.8) we need to verify

$$
\sum_{N_{\text {max }} \sim N_{\text {med }} \sim N} \sum_{L_{\text {max }} \sim L_{\text {med }} \gtrsim N^{2} N_{\text {min }}} \frac{N_{3}<N_{3}>^{s} L_{\text {min }}^{1 / 2} N_{\text {min }}^{1 / 2}}{<N_{1}>^{s}<N_{2}>^{s} L_{1}^{b} L_{2}^{b} L_{3}^{(1-b)-}} \lesssim 1 .
$$

To do so, it follows from symmetry that we are required to handle two cases:

$$
\left\{\begin{array}{l}
N_{1} \sim N_{2} \sim N \& N_{3}=N_{\text {min }} \\
N_{1} \sim N_{3} \sim N \& N_{2}=N_{\text {min }} .
\end{array}\right.
$$

Under the former case, the estimate (3.4) can be further reduced to

$$
\sum_{N_{\max } \sim N_{\text {med }} \sim N} \sum_{L_{\max } \sim L_{\text {med }} \gtrsim N^{2} N_{\min }} \frac{N^{-2 s} N_{\min }<N_{\min }>^{s} L_{\min }^{1 / 2} N_{\min }^{1 / 2}}{L_{\min }^{b} L_{\text {med }}^{b} L_{\text {max }}^{(1-b)-}} \lesssim 1 .
$$

This, after performing the $L$ summations, is reduced to

$$
\sum_{N_{\text {max }} \sim N_{\text {med }} \sim N} \frac{N_{\min }^{3 / 2}<N_{\min }>^{s}}{N^{2 s}\left(N^{2} N_{\text {min }}\right)^{1-}} \lesssim 1,
$$

which is true for $2+2 s>0$. So, (3.4) is valid for $s>-1$. Under the latter case, the estimate (3.4) can be reduced to

$$
\sum_{N_{\text {max }} \sim N_{\text {med }} \sim N} \sum_{L_{\text {max }} \sim L_{\text {med }} \gtrsim N^{2} N_{\text {min }}} \frac{N L_{\min }^{1 / 2} N_{\min }^{1 / 2}}{<N_{\min }>^{s} L_{\min }^{b} L_{\text {med }}^{b} L_{\text {max }}^{(1-b)-}} \lesssim 1 .
$$

However, before performing the $L$ summations, we need to pay a little more attention to the summation of $N_{\min }$. This time, we are required to check

$$
\begin{gathered}
\sum_{N_{\text {max }} \sim N_{\text {med }} \sim N, N_{\text {min }} \leq 1} \sum_{L_{\text {max }} \sim L_{\text {med }} \gtrsim N^{2} N_{\text {min }}} \frac{N N_{\min }^{1 / 2}}{L_{\text {min }}^{b-1 / 2}\left(L_{\text {max }}\right)^{1-}} \\
+\sum_{N_{\text {max }} \sim N_{\text {med }} \sim N, 1 \leq N_{\text {min }} \leq N} \sum_{L_{\text {max }} \sim L_{\text {med }} \gtrsim N^{2} N_{\text {min }}} \frac{N N_{\text {min }}^{1 / 2-s}}{L_{\text {min }}^{b-1 / 2}\left(L_{\text {max }}\right)^{1-}} \lesssim 1,
\end{gathered}
$$

which is obviously true when $s>-3 / 2$. Namely, (3.4) is true for $s>-3 / 2$.

We then show (3.3). Under this circumstance we have $L_{\max } \sim N_{\max }^{2} N_{\min }$ and consequently consider three matters as follows.

The first one is to handle the situation where (2.6) holds. In this case we have $N_{1}, N_{2}, N_{3} \sim N \gtrsim 1$ and so we are required to verify

$$
\sum_{L_{\max } \sim N^{3}} \frac{N^{-s} N}{L_{\text {min }}^{b} L_{\text {med }}^{b} L_{\text {max }}^{(1-b)-}} L_{m i n}^{1 / 2} N^{-1 / 4} L_{\text {med }}^{1 / 4} \lesssim 1 .
$$

Performing the $L$ summations, we are required to check

$$
\frac{N^{3 / 4} N^{-s}}{\left(N^{3}\right)^{(1-b)-}} \lesssim 1,
$$

which is true for $-3 / 4+s+3(1-b)>0$. So, (3.5) is true for both $s>-3 / 4$ and $1 / 2<b<(9+4 s) / 12$.

The second one is to settle the case where (2.7) holds. By symmetry we only need to consider two cases

$$
\left\{\begin{array}{l}
N \sim N_{1} \sim N_{2} \gg N_{3} \& H \sim L_{3} \gtrsim L_{1}, L_{2} ; \\
N \sim N_{2} \sim N_{3} \gg N_{1} \& H \sim L_{1} \gtrsim L_{2}, L_{3} .
\end{array}\right.
$$


For the former: $N \sim N_{1} \sim N_{2} \gg N_{3} \& H \sim L_{3} \gtrsim L_{1}, L_{2}$, we are required by (2.7) to show

$$
\sum_{N_{3} \ll N} \sum_{1 \lesssim L_{1}, L_{2} \lesssim N^{2} N_{3}} \frac{N_{3}<N_{3}>^{s} L_{\min }^{1 / 2} N^{-1}}{N^{2 s} L_{1}^{b} L_{2}^{b} L_{3}^{(1-b)-}}\left(\min \left\{N^{2} N_{3}, \frac{N}{N_{3}} L_{m e d}\right\}\right)^{1 / 2} \lesssim 1 .
$$

Splitting the left-hand side of (3.6) into two pieces $I_{1}$ and $I_{2}$ where

$$
\begin{array}{r}
I_{1}=\sum_{N_{3} \leq 1} \sum_{1 \lesssim L_{1}, L_{2} \lesssim N^{2} N_{3}} \frac{N_{3}<N_{3}>^{s} L_{\min }^{1 / 2} N^{-1} \mathrm{M}}{N^{2 s} L_{1}^{b} L_{2}^{b} L_{3}^{(1-b)-}} ; \\
I_{2}=\sum_{1<N_{3} \ll N} \sum_{1 \lesssim L_{1}, L_{2} \lesssim N^{2} N_{3}} \frac{N_{3}<N_{3}>^{s} L_{\min }^{1 / 2} N^{-1} \mathrm{M}}{N^{2 s} L_{1}^{b} L_{2}^{b} L_{3}^{(1-b)-}} ; \\
\mathrm{M}:=\left(\min \left\{N^{2} N_{3}, \frac{N}{N_{3}} L_{\text {med }}\right\}\right)^{1 / 2} ;
\end{array}
$$

we estimate these two terms separately. The estimate of $I_{1}$ goes like this:

$$
I_{1} \leq \sum_{N_{3} \leq 1} \sum_{1 \lesssim L_{1}, L_{2} \lesssim N^{2} N_{3}} \frac{N_{3} L_{\text {min }}^{1 / 2} N^{-1}\left(\frac{N}{N_{3}} L_{m e d}\right)^{1 / 2}}{N^{2 s} L_{m i n}^{b} L_{m e d}^{b}\left(N^{2} N_{3}\right)^{(1-b)-}} .
$$

Performing the $L$ summation in (3.7), we have that

$$
I_{1} \lesssim \sum_{N_{3} \leq 1} \frac{N_{3}^{1 / 2} N^{-1 / 2}}{N^{2 s}\left(N^{2}\right)^{(1-b)-} N_{3}^{(1-b)-}} \lesssim \frac{N^{-1 / 2}}{N^{2 s}\left(N^{2}\right)^{(1-b)-}} \lesssim 1
$$

if $1 / 2+2 s+2(1-b)>0$. Namely, $I_{1} \lesssim 1$ if $s>-3 / 4$ and $1 / 2<b<5 / 4+s$ are true. The estimate of $I_{2}$ goes like this:

$$
I_{2} \leq \sum_{1<N_{3} \ll N} \sum_{1 \lesssim L_{1}, L_{2} \lesssim N^{2} N_{3}} \frac{N_{3} N_{3}^{s} N^{-1} N^{1 / 2} L_{\text {med }}^{1 / 2} N_{3}^{-1 / 2}}{N^{2 s} L_{\text {min }}^{b-1 / 2} L_{\text {med }}^{b}\left(N^{2} N_{3}\right)^{(1-b)-}} .
$$

Performing the $N_{3}$ summation in (3.8) we obtain that $s-2^{-1}+b<0$ implies

$$
I_{2} \lesssim \sum_{1 \lesssim L_{1}, L_{2} \lesssim N^{3}} \frac{1}{N^{2 s} L_{m i n}^{b-1 / 2} L_{m e d}^{b-1 / 2}\left(N^{2}\right)^{(1-b)-} N^{1 / 2}} \lesssim 1
$$

Of course, this is true if $2 s+2(1-b)+1 / 2>0$. In other words, $I_{2} \lesssim 1$ is valid for

$$
s>-3 / 4 \text { and } \frac{1}{2}<b<\min \left\{5 / 4+s, 2^{-1}-s\right\}
$$

provided $s<1 / 2-b<0$. If $s-1 / 2+b \geq 0$, then

$$
I_{2} \lesssim \sum_{1 \lesssim L_{1}, L_{2} \lesssim N^{3}} \frac{N^{s-1 / 2+b}}{N^{2 s} L_{\text {min }}^{b-1 / 2} L_{\text {med }}^{b-1 / 2}\left(N^{2}\right)^{(1-b)-} N^{1 / 2}} \lesssim 1
$$

is true for

$$
2 s+2(1-b)+1 / 2>s-1 / 2+b .
$$

This means that $I_{2} \lesssim 1$ is true for $s \geq 1 / 2-b$ where $1 / 2<b<7 / 8$. Combining the estimates for $I_{1}$ and $I_{2}$, we obtain the desired estimate (3.6). 
For the latter: $N \sim N_{2} \sim N_{3} \gg N_{1} \& H \sim L_{1} \gtrsim L_{2}, L_{3}$, we see from (2.7) that (3.3) can be established via proving

$$
\sum_{N_{1} \ll N} \sum_{1 \lesssim L_{2}, L_{3} \lesssim N^{2} N_{1}} \frac{N^{1+s} L_{\text {min }}^{1 / 2} N^{-1}\left(\min \left\{H, \frac{N}{N_{1}} L_{\text {med }}\right\}\right)^{1 / 2}}{N^{s}<N_{1}>^{s} L_{2}^{b} L_{3}^{(1-b)-}\left(N^{2} N_{1}\right)^{b}} \lesssim 1 .
$$

Now, writing the left-hand side of (3.9) as $J_{1}+J_{2}$ where

$$
\begin{aligned}
J_{1} & :=\sum_{N_{1} \leq 1} \sum_{1 \lesssim L_{2}, L_{3} \lesssim N^{2} N_{1}} \frac{N^{1+s}}{N^{s} L_{2}^{b} L_{3}^{(1-b)-}\left(N^{2} N_{1}\right)^{b}} L_{\text {min }}^{1 / 2} N_{1}^{1 / 2} ; \\
J_{2} & =\sum_{1<N_{1} \ll N} \sum_{1 \lesssim L_{2}, L_{3} \lesssim N^{2} N_{1}} \frac{N^{1+s}}{N_{1}^{s} N^{s} L_{2}^{b} L_{3}^{(1-b)-}\left(N^{2} N_{1}\right)^{b}} L_{\text {min }}^{1 / 2} N^{-1} N^{3 / 4} L_{\text {med }}^{1 / 4} .
\end{aligned}
$$

In $J_{1}$, we may assume $N_{1} \gtrsim N^{-2}$ - otherwise the summation of $L$ vanishes. Performing the summation of $L$, we get

$$
J_{1} \lesssim \sum_{N^{-2} \lesssim N_{1} \leq 1} \frac{N N_{1}^{\frac{1}{2}-b}}{N^{2 b}} \lesssim \frac{N N^{(-2)(1 / 2-b)}}{N^{2 b}} \lesssim 1 .
$$

If $1 / 2<b<3 / 4$ and $s>-3 / 4$ hold in $J_{2}$, then the summation of $L$ implies

$$
J_{2} \lesssim \sum_{1 \leq N_{1} \ll N} \frac{N^{3 / 4} N_{1}^{-s-b}}{N^{2 b}} \lesssim \frac{N^{3 / 4} N^{\max \{0,-s-b\}}}{N^{2 b}} \lesssim 1 .
$$

Combining the estimates for $J_{1}$ and $J_{2}$, we get the desired estimate (3.9).

The third one is to deal with the case where (2.8) holds. This reduces to

$$
\sum_{N_{\text {max }} \sim N_{\text {med }} \sim N} \sum_{L_{\text {max }} \sim N^{2} N_{\text {min }}} \frac{N_{3}<N_{3}>^{s} L_{\min }^{1 / 2}\left(\min \left\{H, L_{\text {med }}\right\}\right)^{1 / 2}}{<N_{1}>^{s}<N_{2}>^{s} N L_{1}^{b} L_{2}^{b} L_{3}^{(1-b)-}} \lesssim 1 .
$$

To estimate (3.10), by symmetry we need to consider two cases:

$$
\left\{\begin{array}{l}
N_{1} \sim N_{2} \sim N \& N_{3}=N_{\min } \\
N_{1} \sim N_{3} \sim N \& N_{2}=N_{\min }
\end{array}\right.
$$

Regarding the former: $N_{1} \sim N_{2} \sim N \& N_{3}=N_{\min }$, the estimate (3.10) further reduces to

$$
\sum_{\substack{N_{1} \sim N_{2} \sim \sim^{N} \\ N_{3} \ll N}} \sum_{\substack{L_{\text {max }} \sim N^{2} N_{3} \\ N^{2 s} L_{\text {min }}^{b} L_{\text {med }}^{b}\left(N^{2} N_{3}\right)^{(1-b)-}}} L_{\text {min }}^{1 / 2} N^{-1} L_{\text {med }}^{1 / 2} \lesssim 1 .
$$

Performing the $L$ summation, we have

$$
\begin{aligned}
\sum_{N_{3} \ll N} \frac{N_{3}<N_{3}>^{s}}{N^{1+2 s}\left(N^{2}\right)^{(1-b)-} N_{3}^{(1-b)-}}= & \sum_{N_{3} \leq 1} \frac{N_{3}^{b}}{N^{1+2 s}\left(N^{2}\right)^{(1-b)-}} \\
& +\sum_{1<N_{3} \ll N} \frac{N_{3}^{s+b}}{N^{1+2 s}\left(N^{2}\right)^{(1-b)-}} .
\end{aligned}
$$

The first term in the right-hand side of (3.11) is bounded if $s>-1$, while the second term in the right-hand side of (3.11) is less than $\frac{N^{\max \{0, s+b\}}}{N^{1+2 s+2(1-b)-}}$ which is bounded if $s>-1$ and $1 / 2<b<3 / 4$. So, (3.10) is true if $s>-1$. 
Regarding the latter: $N_{1} \sim N_{3} \sim N \& N_{2}=N_{\min }$, the estimate (3.10) can be reduced to

$$
\sum_{\substack{N_{1} \sim N_{3} \sim N \\ N_{2} \ll N}} \sum_{L_{\text {max }} \sim N^{2} N_{2}} \frac{N^{1+s} L_{\min }^{1 / 2} N^{-1}\left(\min \left\{H, L_{\text {med }}\right\}\right)^{1 / 2}}{N^{s}<N_{2}>^{s} L_{\text {min }}^{b} L_{\text {med }}^{b} L_{\text {max }}^{(1-b)-}} \lesssim 1 .
$$

Before performing the $L$ summation, we have to pay a little more attention to the summation of $N_{2}$. The left-hand side of (3.12) is now written as $J_{3}+J_{4}$ where

$$
\begin{array}{r}
J_{3}:=\sum_{N_{2} \leq 1} \sum_{L_{\text {max }} \sim N^{2} N_{2}} \frac{L_{\text {min }}^{1 / 2} L_{\text {med }}^{1 / 2}}{L_{\text {min }}^{b} L_{\text {med }}^{b} L_{\text {max }}^{(1-b)-}} ; \\
J_{4}:=\sum_{1 \leq N_{2} \leq N} \sum_{L_{\text {max }} \sim N^{2} N_{2}} \frac{L_{\text {min }}^{1 / 2} L_{\text {med }}^{1 / 2}}{N_{2}^{s} L_{\text {min }}^{b} L_{\text {med }}^{b} L_{\text {max }}^{(1-b)-}} .
\end{array}
$$

In $J_{3}$ we may assume $N_{2} \gtrsim N^{-2}$ - otherwise the summation of $L$ vanishes. Performing the summation of $L$, we get

$$
J_{3} \lesssim \sum_{N^{-2} \lesssim N_{2} \leq 1} \frac{N_{2}^{(b-1+\epsilon)}}{N^{2(1-b-\epsilon)}} \lesssim \frac{N^{-2(b-1+\epsilon)}}{N^{2(1-b-\epsilon)}} \lesssim 1,
$$

where $\epsilon>0$ is small enough. For $J_{4}$, if $s+1-b \geq 0$, then we always have $J_{4} \lesssim 1$ for any $1 / 2<b \leq 1$. If $s+1-b<0$, we have $J_{4} \lesssim 1$ under $2(1-b)+s+1-b>0$. So, (3.12) is true if $s>-3 / 2$ and $1 / 2<b<(s+3) / 3$. This completes the proof of the first part of Theorem 1.1.

Proof of Theorem 1.1 (ii). Note that

$$
\left\|\partial_{x}(u v)\right\|_{X_{s, b-1, p}} \lesssim\left\|\partial_{x}(u v)\right\|_{X_{s,(b-1)+, p}} .
$$

So it is enough to check that both $s \leq-3 / 4$ and $b \in \mathbb{R}$ cannot imply

$$
\left\|\partial_{x}(u v)\right\|_{X_{s, b-1, p}} \lesssim\|u\|_{X_{s, b, p}}\|v\|_{X_{s, b, p}},
$$

which is equivalent to

$$
\left\|\frac{\iint_{\mathbb{R}^{2}} \frac{f\left(\xi_{1}, \tau_{1}\right) f\left(\xi-\xi_{1}, \tau-\tau_{1}\right)\left(1+\left|\xi_{1}\right|\right)^{-s}\left(1+\left|\tau_{1}-p\left(\xi_{1}\right)\right|\right)^{-b}}{\left(1+\left|\xi-\xi_{1}\right|\right)^{s}\left(1+\left|\tau-\tau_{1}-p\left(\xi-\xi_{1}\right)\right|\right)^{b}} d \xi_{1} d \tau_{1}}{\left(\frac{|\xi|(1+|\xi|)^{s}}{(1+|\tau-p(\xi)|)^{1-b}}\right)^{-1}}\right\|_{L_{\xi, \tau}^{2}\left(\mathbb{R}^{2}\right)} \lesssim\|f\|_{L_{\xi, \tau}^{2}}^{2}\left(\mathbb{R}^{2}\right)^{\prime} .
$$

Case 1: $s<-3 / 4$. On the one hand, given a large natural number $N$ let

$$
f(\xi, \tau)=1_{A}(\xi, \tau)+1_{-A}(\xi, \tau)
$$

where $1_{E}$ stands for the characteristic function of a set $E \subseteq \mathbb{R}^{2}$, and

$$
\begin{gathered}
A=\left\{(\xi, \tau) \in \mathbb{R}^{2}: N \leq \xi \leq N+N^{-1 / 2} \text { and }|\tau-p(\xi)| \leq 1\right\} ; \\
-A=\left\{(\xi, \tau) \in \mathbb{R}^{2}:(-\xi,-\tau) \in A\right\} .
\end{gathered}
$$

See also [17] for the definitions of $A$ and $-A$ in the case $p(\xi)=\xi^{3}$. Clearly, we have

$$
\|f\|_{L_{\xi, \tau}^{2}\left(\mathbb{R}^{2}\right)} \lesssim N^{-1 / 4} \text {. }
$$


Note that $A$ contains a rectangle with $(N, p(N))$ as a vertex, with dimensions $10^{-2} N^{-2} \times N^{-1 / 2}$, and with longest side pointing in the $\left(1, p^{\prime}(N)\right)$-direction where $p^{\prime}(N)=3 \beta N^{2}-2 \alpha N+\gamma$. So

$$
|(f * f)(\xi, \tau)| \gtrsim N^{-1 / 2} 1_{R}(\xi, \tau)
$$

where $R$ is the rectangle centered at the origin with dimensions $\sim N^{-2} \times N^{-1 / 2}$ and longest side pointing in the $\left(1, p^{\prime}(N)\right)$-direction. Consequently, (3.14) implies

$$
N^{-2 s} N^{-1 / 2} N^{3(b-1) / 2} N^{-1 / 2} N^{-1 / 4} \lesssim N^{-1 / 2}
$$

and thus $b \leq 1 / 2$. On the other hand, we also show $b>1 / 2$. To this end, we apply polarization and duality to obtain that (3.14) amounts to

$$
\left|\iint_{\mathbb{R}^{2}} \frac{\iint_{\mathbb{R}^{2}} \frac{f\left(\xi_{1}, \tau_{1}\right) f\left(\xi-\xi_{1}, \tau-\tau_{1}\right)<\xi_{1}>^{-s}<\tau_{1}-p\left(\xi_{1}\right)>^{-b}}{<\xi-\xi_{1}>^{s}<\tau-\tau_{1}-p\left(\xi-\xi_{1}\right)>^{b}} d \xi_{1} d \tau_{1}}{\left(\frac{\xi<\xi>^{s} g(\xi, \tau)}{<\tau-p(\xi)>^{1-b}}\right)^{-1}}\right| \lesssim\|f\|_{L_{\xi, \tau}^{2}\left(\mathbb{R}^{2}\right)}^{2}\|g\|_{L_{\xi, \tau}^{2}\left(\mathbb{R}^{2}\right)},
$$

which is equivalent to

$$
\left\|\frac{\iint_{\mathbb{R}^{2}} \frac{g(\xi, \tau) h\left(\xi-\xi_{1}, \tau-\tau_{1}\right) \xi<\xi>^{s}}{\left\langle\tau-p(\xi)>^{1-b}<\xi-\xi_{1}>^{s}<\tau-\tau_{1}-p\left(\xi-\xi_{1}>^{b}\right.\right.} d \xi d \tau}{<\xi_{1}>^{s}<\tau_{1}-p\left(\xi_{1}\right)>^{b}}\right\|_{L_{\xi_{1}, \tau_{1}}^{2}\left(\mathbb{R}^{2}\right)} \lesssim\|g\|_{L_{\xi, \tau}^{2}\left(\mathbb{R}^{2}\right)}\|h\|_{L_{\xi, \tau}^{2}\left(\mathbb{R}^{2}\right)} .
$$

Now, if

$$
g(\xi, \tau)=1_{A}(\xi, \tau) \text { and } \quad h(\xi, \tau)=1_{B}(\xi, \tau)
$$

where

$$
B=\left\{(\xi, \tau) \in \mathbb{R}^{2}:-N+\frac{1}{2 \sqrt{N}} \leq \xi \leq-N+\frac{3}{4 \sqrt{N}} \quad \text { and } \quad|\tau-p(\xi)| \leq 1\right\} .
$$

Estimating the left-hand side of (3.15) via the domain determined by

$$
\left|4 \tau_{1}-p\left(\xi_{1}\right)\right| \leq 4^{-1} \quad \text { and } \quad 2 N-\frac{11}{16 \sqrt{N}} \leq \xi_{1} \leq 2 N-\frac{9}{16 \sqrt{N}}
$$

we find

$$
N^{1 / 4} N^{-s} N^{-3 b} \lesssim N^{-1 / 2}
$$

whence reaching $b>1 / 2$ by $s<-3 / 4$. Of course, we have a contradiction.

Case 2: $s=-3 / 4$. From the argument for Case 1 we see that (3.13) enforces $b=1 / 2$. Without loss of generality we may assume the natural numbers $N$ and $m$ are so big that $4^{m+1} \ll N$. With this assumption and that construction in [21, Proof of Theorem 1 (i)] in mind we define a sequence of sets as follows:

$$
A_{j}=\left\{(\xi, \tau) \in \mathbb{R}^{2}: N \leq|\xi| \leq N+\sqrt{4^{m+1} / N} \text { and } 4^{j} \leq|\tau-p(\xi)|<4^{j+1}\right\}
$$

for $j=0,1, \ldots, m-1$; and $A_{m}$ is the union of two parallelograms with two groups of vertices

$$
\left\{\begin{array}{l}
\left(N, p(N)-4^{m}\right) \\
\left(N, p(N)-4^{m+1}\right) \\
\left(N+\sqrt{4^{m+1} / N}, p(N)+p^{\prime}(N) \sqrt{4^{m+1} / N}-4^{m}\right) \\
\left(N+\sqrt{4^{m+1} / N}, p(N)+p^{\prime}(N) \sqrt{4^{m+1} / N}-4^{m+1}\right)
\end{array}\right.
$$


and

$$
\left\{\begin{array}{l}
\left(-N, p(-N)+4^{m}\right) ; \\
\left(-N, p(-N)+4^{m+1}\right) ; \\
\left(-N-\sqrt{4^{m+1} / N}, p(-N)-p^{\prime}(-N) \sqrt{4^{m+1} / N}+4^{m}\right) ; \\
\left(-N-\sqrt{4^{m+1} / N}, p(-N)-p^{\prime}(-N) \sqrt{4^{m+1} / N}+4^{m+1}\right) .
\end{array}\right.
$$

Also, we set $R$ be the region comprising two parallelograms similar to those two parallelograms making $A_{m}$, but with area being of a quarter of $A_{m}$ 's area, with two centers

$$
\left(-\frac{7}{12} \sqrt{\frac{4^{m+1}}{N}}, 0\right) ; \quad\left(\frac{7}{12} \sqrt{\frac{4^{m+1}}{N}}, 0\right),
$$

and with the longest sides parallel to the point

$$
\left(\sqrt{\frac{4^{m+1}}{N}}, p^{\prime}(N) \sqrt{\frac{4^{m+1}}{N}}\right) .
$$

Next, given $\left\{a_{j}\right\}_{j=0}^{m}$, a finite sequence of positive numbers let $f$ be the function on $\mathbb{R}^{2}$ decided by its Fourier transform:

$$
\hat{f}=N \sum_{j=0}^{m} 4^{-j-\frac{m}{4}} a_{j} 1_{A_{j}} .
$$

Then a straightforward computation (cf. [21, (2.6)]) gives

$$
\hat{f} * \hat{f} \geq N^{2} a_{m}\left(\sum_{j=0}^{m} 4^{-j-\frac{3 m}{2}} a_{j} 1_{A_{j}}\right) * 1_{A_{m}} .
$$

Applying this last inequality and noticing the following simple facts (cf. [21, (2.1)$(2.5)])$ :

$$
\begin{aligned}
& \operatorname{Area}\left(A_{j}\right) \sim 4^{j+\frac{m}{2}} N^{-1 / 2} \quad \text { for } j=0,1, \ldots, m-1 ; \quad \operatorname{Area}\left(A_{m}\right) \sim 4^{3 m / 2} N^{-1 / 2}, \\
& \quad \operatorname{Area}(R) \sim 4^{3 m / 2} N^{-1 / 2} ; \quad \operatorname{Area}\left(A_{j}\right) 1_{R} \lesssim 1_{A_{j}} * 1_{A_{m}} \quad \text { for } j=0,1, \ldots, m,
\end{aligned}
$$

and

$$
j \neq k \Longrightarrow A_{j} \cap A_{k}=\emptyset
$$

we get two groups of inequalities:

$$
\begin{aligned}
\left\|\partial_{x}\left(f^{2}\right)\right\|_{X_{-3 / 4,-1 / 2, p}} & =\left\||\xi|(1+|\xi|)^{-3 / 4}(1+|\tau-p(\xi)|)^{-1 / 2} \hat{f} * \hat{f}\right\|_{L_{\xi, \tau}^{2}\left(\mathbb{R}^{2}\right)} \\
& \gtrsim \frac{\left(\iint_{R}\left(\sum_{j=0}^{m} 4^{-j-3 m / 2} a_{j}\left(1_{A_{j}} * 1_{A_{m}}\right)\right)^{2} d \xi d \tau\right)^{1 / 2}}{\left(4^{(m+1) / 4} N^{3 / 4} a_{m}\right)^{-1}} \\
& \gtrsim a_{m} \sum_{j=0}^{m} a_{j}
\end{aligned}
$$

and

$$
\begin{aligned}
\left\|\frac{(1+|\tau-p(\xi)|)^{1 / 2} \hat{f}}{(1+|\xi|)^{3 / 4}}\right\|_{L_{\xi, \tau}^{2}\left(\mathbb{R}^{2}\right)} & =\left(\iint_{\mathbb{R}^{2}} \frac{\left.\sum_{j=0}^{m} \frac{a_{j}^{2} 1_{A_{j}}}{\left(\frac{N^{2}(1+|\tau-p(\xi)|)}{(1+|\xi|)^{3 / 2}}\right)^{-1}} d \xi d \tau\right)^{1 / 2}}{}\right. \\
& \lesssim\left(\sum_{j=0}^{m} a_{j}^{2}\right)^{1 / 2}
\end{aligned}
$$


thereby reaching via (3.13)

$$
a_{m} \sum_{j=0}^{m} \lesssim a_{j} \sum_{j=0}^{m} a_{j}^{2}
$$

However (3.16) is not always true. In fact, if

$$
a_{j}=(1+j)^{-1} \quad \text { for } \quad j=0,1, \ldots, m-1 \quad \text { and } \quad a_{m}=1,
$$

then one has a contradictory inequality:

$$
\sum_{j=1}^{m+1} j^{-1} \lesssim \sum_{j=1}^{m+1} j^{-2} \quad \text { as } \quad m \rightarrow \infty .
$$

Therefore, the proof of Theorem 1.2 (ii) is complete.

\section{Sharp Local Well/ILL-POSEDNESS}

This section is devoted to verifying Theorem 1.2 .

Proof of Theorem 1.2 (i). We start with a few notations. Denote by $W(t)$ the unitary group generating the solution of the Cauchy problem for the linear equation

$$
\left\{\begin{array}{l}
\partial_{t} v-\gamma \partial_{x} v+\alpha \mathcal{H} \partial_{x}^{2} v+\beta \partial_{x}^{3} v=0, \quad(x, t) \in \mathbb{R} \times \mathbb{R} \\
v(x, 0)=v_{0}(x), \quad x \in \mathbb{R} .
\end{array}\right.
$$

That is,

$$
v(x, t)=W(t) v_{0}(x)=S_{t} * v_{0}(x)
$$

where

$$
\widehat{S}_{t}=e^{i t p(\xi)} \quad \text { or } \quad S_{t}(x)=\int_{\mathbb{R}} e^{i(x \xi+t p(\xi))} d \xi \quad \text { with } \quad p(\xi)=\beta \xi^{3}-\alpha \xi|\xi|+\gamma \xi .
$$

Let $\psi \in C_{0}^{\infty}(\mathbb{R})$ be a standard bump function such that $\psi(t) \equiv 1$ if $|t|<1$ and $\psi(t) \equiv 0$ if $|t|>2$. Consider the following integral equation

$$
u(x, t)=\psi(t) W(t) u_{0}(x)-2 \psi\left(\delta^{-1} t\right) \int_{0}^{t} W\left(t-t^{\prime}\right) u\left(x, t^{\prime}\right) \partial_{x} u\left(x, t^{\prime}\right) d t^{\prime}
$$

for $0<\delta<1$. Denote the right-hand side by $\mathcal{T}(u)(x, t)$. The goal is to show that $\mathcal{T}(u)$ is a contraction map from $Y$ to itself, where

$$
Y=\left\{u \in X_{s, b, p}:\|u\|_{X_{s, b, p}} \leq 2 c_{0}\left\|u_{0}\right\|_{H^{s}}\right\},
$$

where $c_{0}$ is the constant appeared in the following linear estimates - under $\alpha, 0 \neq$ $\beta, \gamma \in \mathbb{R}$, one has that for $1 / 2<b \leq 1$,

$$
\left\|\psi\left(\delta^{-1} t\right) W(t) u_{0}(x)\right\|_{X_{s, b, p}} \leq c_{0} \delta^{(1-2 b) / 2}\left\|u_{0}\right\|_{H^{s}}
$$

and for $b^{\prime}+1 \geq b \geq 0 \geq b^{\prime}>-1 / 2$,

$$
\left\|\psi\left(\delta^{-1} t\right) \int_{0}^{t} W\left(t-t^{\prime}\right) f\left(x, t^{\prime}\right) d t^{\prime}\right\|_{X_{s, b, p}} \leq c_{0} \delta^{1+b^{\prime}-b}\|f\|_{X_{s, b^{\prime}, p}} .
$$

Inequality (4.1) follows from Kenig-Ponce-Vega [16, and inequality (4.2) follows from the inhomogeneous linear equation version stated in [11, Lemma 2.1] and [12, Lemma 1.9]. 
If $u \in Y$, then $b^{\prime}$ is taken to be $b-1+\sigma$ where $\sigma>0$ is small enough to ensure that $0 \geq b^{\prime}>-1 / 2$, and hence a combined application of (4.1), (4.2) and Theorem 1.1 (i) yields

$$
\begin{aligned}
\|\mathcal{T}(u)\|_{X_{s, b, p}} & \leq\left\|\psi(t) W(t) u_{0}(x)\right\|_{X_{s, b, p}} \\
& +2\left\|\psi\left(\delta^{-1} t\right) \int_{0}^{t} W\left(t-t^{\prime}\right) u\left(x, t^{\prime}\right) \partial_{x} u\left(x, t^{\prime}\right) d t^{\prime}\right\|_{X_{s, b, p}} \\
& \leq c_{0}\left\|u_{0}\right\|_{H^{s}}+2 c_{0} \delta^{\sigma}\left\|u \partial_{x} u\right\|_{X_{s, b^{\prime}, p}} \\
& \leq c_{0}\left\|u_{0}\right\|_{H^{s}}+c_{0} c \delta^{\sigma}\|u\|_{X_{s, b, p}}^{2} \\
& \leq c_{0}\left\|u_{0}\right\|_{H^{s}}+4 c_{0}^{3} c \delta^{\sigma}\left\|u_{0}\right\|_{H^{s}}^{2}
\end{aligned}
$$

If $\delta>0$ is such a small number that $4 c_{0}^{2} c \delta^{\sigma}\left\|u_{0}\right\|_{H^{s}} \leq 1 / 2$, then one has $\mathcal{T}(u) \in Y$. Also, if $u, v \in Y$,

$$
\begin{aligned}
\|\mathcal{T}(u)-\mathcal{T}(v)\|_{X_{s, b, p}} & \leq c_{0} c \delta^{\sigma}\|u+v\|_{X_{s, b, p}}\|u-v\|_{X_{s, b, p}} \\
& \leq 4 c_{0}^{2} c \delta^{\sigma}\left\|u_{0}\right\|_{H^{s}}\|u-v\|_{X_{s, b, p}} \\
& \leq 2^{-1}\|u-v\|_{X_{s, b, p}} .
\end{aligned}
$$

Therefore $\mathcal{T}$ is a contraction mapping on $Y$. By the classical Banach fixed point theorem, there exists a unique solution $u \in Y$ such that

$$
u(x, t)=\psi(t) W(t) u_{0}(x)-2 \psi\left(\delta^{-1} t\right) \int_{0}^{t} W\left(t-t^{\prime}\right) u\left(x, t^{\prime}\right) \partial_{x} u\left(t^{\prime}\right) d t^{\prime}
$$

Choosing $T=2^{-1} \delta$, we have that $t \in[0, T]$ implies $\psi(t)=1$ and so that $u(x, t)$ solves the integral equation associated to the Cauchy problem (1.2).

Next, we verify the persistence property $u \in C\left([0, T], H^{s}(\mathbb{R})\right)$ and the continuous dependence of the solution upon the data. Clearly, the former follows directly from [24, Corollary 2.1] which gives

$$
\sup _{t \in[0, T]}\|u(\cdot, t)\|_{H^{s}} \lesssim\|u\|_{X_{s, b, p}} .
$$

As to the latter, we apply (4.1)-(4.2), 24, Corollary $2.1 \&$ Lemma 2.64] (with $\eta$ being a Schwartz function on $\mathbb{R}$, e.g., $\eta=\psi$ as above) and Theorem[1.1(i) to obtain 
that if $0 \leq t_{0}<t \leq T$ and $t-t_{0} \leq \Delta t$ then

$$
\begin{aligned}
\left\|u(\cdot, t)-u\left(\cdot, t_{0}\right)\right\|_{H^{s}} & \lesssim\left\|W\left(t-t_{0}\right) u\left(\cdot, t_{0}\right)-u\left(\cdot, t_{0}\right)\right\|_{H^{s}} \\
& \quad+\left\|\int_{t_{0}}^{t} W\left(t-t^{\prime}\right) \eta^{2}\left(\frac{t^{\prime}-t_{0}}{\Delta t}\right) \partial_{x}\left(u^{2}\left(\cdot, t^{\prime}\right)\right) d t^{\prime}\right\|_{H^{s}} \\
& \lesssim\left\|W\left(t-t_{0}\right) u\left(\cdot, t_{0}\right)-u\left(\cdot, t_{0}\right)\right\|_{H^{s}} \\
& \quad+\left\|\int_{t_{0}}^{t} W\left(t-t^{\prime}\right) \eta^{2}\left(\frac{t^{\prime}-t_{0}}{\Delta t}\right) \partial_{x}\left(u^{2}\left(\cdot, t^{\prime}\right)\right) d t^{\prime}\right\|_{X_{s, b, p}} \\
& \lesssim\left\|W\left(t-t_{0}\right) u\left(\cdot, t_{0}\right)-u\left(\cdot, t_{0}\right)\right\|_{H^{s}} \\
& \quad+\left\|\eta^{2}\left(\frac{t^{\prime}-t_{0}}{\Delta t}\right) \partial_{x}\left(u^{2}\left(\cdot, t^{\prime}\right)\right)\right\|_{X_{s, b-1, p}} \\
\lesssim & \quad+W\left(t-t_{0}\right) u\left(\cdot, t_{0}\right)-u\left(\cdot, t_{0}\right) \|_{H^{s}} \\
& \quad+(\Delta t)^{0+}\left\|\partial_{x}\left(u^{2}\left(\cdot, t^{\prime}\right)\right)\right\|_{X_{s,(b-1)+, p}} \\
\lesssim & \left\|W\left(t-t_{0}\right) u\left(\cdot, t_{0}\right)-u\left(\cdot, t_{0}\right)\right\|_{H^{s}}+(\Delta t)^{0+}\|u\|_{X_{s, b, p}}^{2} \\
= & o(1) \quad \text { as } \Delta t \rightarrow 0
\end{aligned}
$$

giving the persistence property.

To close the argument we need to demonstrate that the uniqueness of the solution to (1.2). To this end, for $\tau>0$ let

$$
\|u\|_{X_{s, b, p}^{\tau}}=\inf \left\{\|v\|_{X_{s, b, p}}: v \in X_{s, b, p} \quad \text { and } \quad v(\cdot, t)=u(\cdot, t) \quad \text { for } \quad t \in[0, \tau]\right\} .
$$

Clearly, if $\left\|u_{1}-u_{2}\right\|_{X_{s, b, p}^{\tau}}=0$, then $u_{1}(\cdot, t)=u_{2}(\cdot, t)$ in $H^{s}(\mathbb{R})$ for $t \in[0, \tau]$. Suppose now that $u_{1}$ is the solution on $[0, T]$ obtained by the fixed point theorem as above and $u_{2}$ is a solution of the integral equation associated to (1.2) with the same initial data $u_{0}$. Without loss of generality, we may assume that there is a constant $M>1$ such that

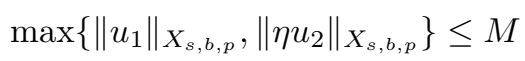

where $\eta$ is the above-appeared bump function on $\mathbb{R}$, and also $T \in(0,1)$. Then for $0<T^{*}<T$ one has

$$
\eta(t) u_{2}(x, t)=\eta(t) W(t) u_{0}(x)-\eta(t) \int_{0}^{t} W\left(t-t^{\prime}\right) \eta\left(\frac{t^{\prime}}{T^{*}}\right) \eta^{2}\left(t^{\prime}\right) \partial_{x}\left(u_{2}^{2}\left(x, t^{\prime}\right)\right) d t^{\prime}
$$

where $(x, t) \in \mathbb{R} \times\left[0, T^{*}\right]$.

For any $\epsilon>0$ there exists $w \in X_{s, b, p}$ such that $t \in\left[0, T^{*}\right]$ implies $w(x, t)=u_{1}(x, t)-\eta(t) u_{2}(x, t) \quad$ and $\quad\|w\|_{X_{s, b, p}} \leq\left\|u_{1}(x, t)-\eta(t) u_{2}(x, t)\right\|_{X_{s, b, p}^{T^{*}}}+\epsilon$.

So if

$$
\tilde{w}(x, t)=-\eta(t) \int_{0}^{t} W\left(t-t^{\prime}\right) \eta\left(\frac{t^{\prime}}{T^{*}}\right) \partial_{x}\left(w\left(x, t^{\prime}\right) u_{1}\left(x, t^{\prime}\right)+\eta\left(t^{\prime}\right) w\left(x, t^{\prime}\right) u_{2}\left(x, t^{\prime}\right)\right) d t^{\prime},
$$

then

$$
\tilde{w}(x, t)=w(x, t)=u_{1}(x, t)-\eta(t) u_{2}(x, t) \quad \text { for } \quad t \in\left[0, T^{*}\right] .
$$


According to the linear estimates (4.1)-(4.2) and Theorem 1.1 (i), for $-1 / 2<b^{\prime}=$ $b-1+\sigma \leq 0$ (with $0<\sigma$ being small enough) we have

$$
\begin{aligned}
\| u_{1}(x, t) & -\eta(t) u_{2}(x, t) \|_{X_{s, b, p}^{T *}} \\
& \leq\|\tilde{w}\|_{X_{s, b, p}} \\
& \lesssim\left(T^{*}\right)^{\sigma} \| \eta\left(t^{\prime} / T^{*}\right) \partial_{x}\left(w\left(x, t^{\prime}\right) u_{1}\left(x, t^{\prime}\right)+\eta\left(t^{\prime}\right) w\left(x, t^{\prime}\right) u_{2}\left(x, t^{\prime}\right) \|_{X_{s, b^{\prime}, p}}\right. \\
& \lesssim\left(T^{*}\right)^{\sigma}\left(\|w\|_{X_{s, b, p}}\left\|u_{1}\right\|_{X_{s, b, p}}+\|w\|_{X_{s, b, p}}\left\|\eta u_{2}\right\|_{X_{s, b, p}}\right) \\
& \lesssim M\left(T^{*}\right)^{\sigma}\|w\|_{X_{s, b, p}},
\end{aligned}
$$

which produces a constant $c_{1}>0$ such that

$$
\left\|u_{1}(x, t)-\eta(t) u_{2}(x, t)\right\|_{X_{s, b, p}^{T^{*}}} \leq c_{1} M\left(T^{*}\right)^{\sigma}\|w\|_{X_{s, b, p}} .
$$

If $T^{*} \leq\left(2 c_{1} M\right)^{-1 / \sigma}$ then

$\left\|u_{1}(x, t)-\eta(t) u_{2}(x, t)\right\|_{X_{s, b, p}^{T^{*}}} \leq 2^{-1}\|w\|_{X_{s, b, p}} \leq 2^{-1}\left(\left\|u_{1}(x, t)-\eta(t) u_{2}(x, t)\right\|_{X_{s, b, p}^{T^{*}}}+\epsilon\right)$

and hence

$$
\left\|u_{1}(x, t)-\eta(t) u_{2}(x, t)\right\|_{X_{s, b, p}^{T *}} \leq 2 \epsilon .
$$

Since $\epsilon>0$ is arbitrary, the last inequality yields that $u_{1}(\cdot, t)=u_{2}(\cdot, t)$ for all $t \in\left[0, T^{*}\right]$. Continuing this process, we achieve the uniqueness assertion on $[0, T]$.

Proof of Theorem 1.2 (ii). Under $s<-3 / 4$ for contradiction we assume that the solution map

$$
u_{0} \in H^{s}(\mathbb{R}) \mapsto u \in C\left([0, T] ; H^{s}(\mathbb{R})\right)
$$

is continuous at zero. According to Bejenaru-Tao's [4, Theorem 3 \& Proposition 1] - a general principle for well-posedness, we must have

$$
\sup _{t \in[0, T]}\left\|A_{3}(f)\right\|_{H^{s}} \lesssim\|f\|_{H^{s}}^{3} \quad \text { for all } \quad f \in H^{s}(\mathbb{R}),
$$

where

$$
\begin{gathered}
A_{3}(f)(x, t)=\int_{\mathbb{R}} e^{i x \xi}\left(\int_{0}^{t} \frac{(i \xi)\left(\int_{\mathbb{R}} \widehat{A_{1}(f)}\left(\xi^{\prime}, t^{\prime}\right) \widehat{A_{2}(f)}\left(\xi-\xi^{\prime}, t^{\prime}\right) d \xi^{\prime}\right)}{e^{i\left(t^{\prime}-t\right) p(\xi)}} d t^{\prime}\right) d \xi \\
A_{2}(f)(x, t)=\int_{\mathbb{R}} \frac{\left(\int_{\mathbb{R}} \frac{\left.\left(\int_{0}^{t} e^{i t^{\prime}\left(p\left(\xi_{1}\right)+p\left(\xi_{2}\right)-p\left(\xi_{1}+\xi_{2}\right)\right.}\right) d t^{\prime}\right)}{\left(e^{i t p\left(\xi_{1}+\xi_{2}\right)}\left(i\left(\xi_{1}+\xi_{2}\right)\right) \hat{f}\left(\xi_{1}\right) \hat{f}\left(\xi_{2}\right)\right)^{-1}} d \xi_{1}\right)}{e^{-i x\left(\xi_{1}+\xi_{2}\right)}} d \xi_{2} \\
A_{1}(f)(x, t)=\int_{\mathbb{R}} e^{i t p(\xi)+i x \xi} \hat{f}(\xi) d \xi
\end{gathered}
$$

In the definition of $A_{3}$, the Fourier transform is taken over the spatial variable.

Motivated by the selection of a test function in [7] and [25] we choose an $H^{s}(\mathbb{R})$ function $f$ with

$$
\|f\|_{H^{s}} \sim 1 \quad \text { and } \quad \hat{f}(\xi)=r^{-1 / 2} N^{-s} 1_{[-r, r]}(|\xi|-N),
$$

where $r=(\sqrt{N} \log N)^{-1}, N>0$ is sufficiently large, and $1_{E}$ stands for the characteristic function of a set $E \subseteq \mathbb{R}$. 
The key issue is to control $\left\|A_{3}(f)\right\|_{H^{s}}$ from below. To proceed, we make the following estimates:

$$
A_{1}(f)(x, t) \sim r^{-1 / 2} N^{-s} \int_{|\xi \pm N|<r} e^{i t p(\xi)+i x \xi} d \xi
$$

and

$$
A_{2}(f)(x, t) \sim F_{1}(x, t)-F_{2}(x, t)
$$

where

$$
F_{1}(x, t)=r^{-1} N^{-2 s} \iint_{\max _{j=1,2}\left|\xi_{j} \pm N\right|<r} \frac{\left(\xi_{1}+\xi_{2}\right) e^{i x\left(\xi_{1}+\xi_{2}\right)+i t\left(p\left(\xi_{1}\right)+p\left(\xi_{2}\right)\right)}}{p\left(\xi_{1}\right)+p\left(\xi_{2}\right)-p\left(\xi_{1}+\xi_{2}\right)} d \xi_{1} d \xi_{2}
$$

and

$$
F_{2}(x, t)=r^{-1} N^{-2 s} \iint_{\max _{j=1,2}\left|\xi_{j} \pm N\right|<r} \frac{\left(\xi_{1}+\xi_{2}\right) e^{i x\left(\xi_{1}+\xi_{2}\right)+i t\left(p\left(\xi_{1}+\xi_{2}\right)\right)}}{p\left(\xi_{1}\right)+p\left(\xi_{2}\right)-p\left(\xi_{1}+\xi_{2}\right)} d \xi_{1} d \xi_{2} .
$$

The contribution of $F_{1}$ to $A_{3}(f)$ is comparable with

$$
r^{-3 / 2} N^{-3 s} \iiint_{\max _{j=1,2,3}\left|\xi_{j} \pm N\right|<r} \frac{Q_{1}\left(\xi_{1}, \xi_{2}, \xi_{3}\right) Q_{2}\left(\xi_{1}, \xi_{2}, \xi_{3}\right)}{e^{-i x\left(\xi_{1}+\xi_{2}+\xi_{3}\right)-i t\left(p\left(\xi_{1}+\xi_{2}+\xi_{3}\right)\right)}} d \xi_{1} d \xi_{2} d \xi_{3},
$$

where

$$
Q_{1}\left(\xi_{1}, \xi_{2}, \xi_{3}\right):=\frac{\left(\xi_{1}+\xi_{2}+\xi_{3}\right)\left(\xi_{2}+\xi_{3}\right)}{p\left(\xi_{2}\right)+p\left(\xi_{3}\right)-p\left(\xi_{2}+\xi_{3}\right)}
$$

and

$$
Q_{2}\left(\xi_{1}, \xi_{2}, \xi_{3}\right):=\frac{e^{i t\left(p\left(\xi_{1}\right)+p\left(\xi_{2}\right)+p\left(\xi_{3}\right)-p\left(\xi_{1}+\xi_{2}+\xi_{3}\right)\right)}-1}{p\left(\xi_{1}\right)+p\left(\xi_{2}\right)+p\left(\xi_{3}\right)-p\left(\xi_{1}+\xi_{2}+\xi_{3}\right)}
$$

Setting

$$
\theta=p\left(\xi_{1}\right)+p\left(\xi_{2}\right)+p\left(\xi_{3}\right)-p\left(\xi_{1}+\xi_{2}+\xi_{3}\right) \quad \text { and } \quad-\xi_{4}=\xi_{1}+\xi_{2}+\xi_{3},
$$

we employ $p(\xi)=\beta \xi^{3}-\alpha \xi|\xi|+\gamma \xi$ to get

$$
\theta=\beta\left(\xi_{1}^{3}+\xi_{2}^{3}+\xi_{3}^{3}+\xi_{4}^{3}\right)-\alpha\left(\xi_{1}\left|\xi_{1}\right|+\xi_{2}\left|\xi_{2}\right|+\xi_{3}\left|\xi_{3}\right|+\xi_{4}\left|\xi_{4}\right|\right) .
$$

By symmetry we may assume that $\left|\xi_{1}\right| \geq\left|\xi_{2}\right| \geq\left|\xi_{3}\right| \geq\left|\xi_{4}\right|$ and further $\xi_{1} \geq 0$ thanks to $\sum_{j=1}^{4} \xi_{j}=0$, and consequently consider two cases according to the signs of $\xi_{1}, \xi_{2}, \xi_{3}$ and $\xi_{4}$.

Case 1: $(+,-,-,-)$. In this case we have

$$
\begin{aligned}
\theta & =3 \beta\left(\xi_{1}+\xi_{4}\right)\left(\xi_{2}+\xi_{4}\right)\left(\xi_{3}+\xi_{4}\right)-\alpha\left(\xi_{1}^{2}-\xi_{2}^{2}-\xi_{3}^{2}-\xi_{4}^{2}\right) \\
& =3 \beta\left(\xi_{1}+\xi_{4}\right)\left(\xi_{2}+\xi_{4}\right)\left(\xi_{3}+\xi_{4}\right)-\alpha\left(\left(\xi_{1}-\xi_{2}\right)\left(\xi_{1}+\xi_{2}\right)-\left(\xi_{3}+\xi_{4}\right)^{2}+2 \xi_{3} \xi_{4}\right) \\
& =3 \beta\left(\xi_{1}+\xi_{4}\right)\left(\xi_{2}+\xi_{4}\right)\left(\xi_{3}+\xi_{4}\right)-\alpha\left(\left(\xi_{3}+\xi_{4}\right)\left(\xi_{2}-\xi_{1}-\xi_{3}-\xi_{4}\right)+2 \xi_{3} \xi_{4}\right) \\
& =3 \beta\left(\xi_{1}+\xi_{4}\right)\left(\xi_{2}+\xi_{4}\right)\left(\xi_{3}+\xi_{4}\right)-\alpha\left(\left(\xi_{3}+\xi_{4}\right)\left(2 \xi_{2}\right)+2 \xi_{3} \xi_{4}\right),
\end{aligned}
$$

whence finding

$$
\theta \sim\left\{\begin{array}{l}
\xi_{1} \xi_{2} \xi_{3} \text { if }\left|\xi_{4}\right| \ll\left|\xi_{1}\right| \\
\xi_{2}^{2} \xi_{3} \text { if }\left|\xi_{4}\right| \sim\left|\xi_{1}\right|
\end{array}\right.
$$


Case 2: $(+,-,-,+)$. In this case we have

$$
\begin{aligned}
\theta & =\beta\left(\xi_{1}^{3}+\xi_{2}^{3}+\xi_{3}^{3}+\xi_{4}^{3}\right)-\alpha\left(\xi_{1}^{2}-\xi_{2}^{2}-\xi_{3}^{2}-\xi_{4}^{2}\right) \\
& =3 \beta\left(\xi_{1}+\xi_{4}\right)\left(\xi_{2}+\xi_{4}\right)\left(\xi_{3}+\xi_{4}\right)-2 \alpha\left(\xi_{3}+\xi_{4}\right)\left(\xi_{2}+\xi_{4}\right) \\
& =3 \beta\left(\xi_{2}+\xi_{4}\right)\left(\xi_{3}+\xi_{4}\right)\left(\xi_{1}+\xi_{4}-\frac{2 \alpha}{3 \beta}\right) \\
& \sim\left(\xi_{2}+\xi_{4}\right)\left(\xi_{3}+\xi_{4}\right) \xi_{1} \\
& \sim\left(\xi_{1}+\xi_{3}\right)\left(\xi_{1}+\xi_{2}\right) \xi_{1} .
\end{aligned}
$$

Thus

$$
|\theta| \sim N^{3} \quad \text { or } \quad|\theta| \lesssim r^{2} N \sim(\log N)^{-2} .
$$

This tells us that the major contribution to (4.3) is obtained via

$$
G_{1}(x, t)=\frac{\iiint_{\max _{j=1,2,3}\left\{\left|\xi_{j} \pm N\right|\right\}<r,|\theta| \lesssim r^{2} N} \frac{Q_{1}\left(\xi_{1}, \xi_{2}, \xi_{3}\right)}{\left.e^{-i\left(x\left(\xi_{1}+\xi_{2}+\xi_{3}\right)+t p\left(\xi_{1}+\xi_{2}+\xi_{3}\right)\right.}\right)} d \xi_{1} d \xi_{2} d \xi_{3}}{r^{3 / 2} N^{3 s}}
$$

with

$$
\left\|G_{1}\right\|_{H^{s}} \sim r N^{-2 s-1} \sim N^{-2 s-3 / 2}(\log N)^{-1} .
$$

On the other hand, the contribution of $F_{2}$ to $A_{3}(f)$ is comparable with

$$
G_{2}(x, t)=\frac{\iiint_{\max _{j=1,2,3}\left|\xi_{j} \pm N\right|<r} \frac{Q_{1}\left(\xi_{1}, \xi_{2}, \xi_{3}\right) Q_{3}\left(\xi_{1}, \xi_{2}, \xi_{3}\right)}{\left.e^{-i\left(x\left(\xi_{1}+\xi_{2}+\xi_{3}\right)+t p\left(\xi_{1}+\xi_{2}+\xi_{3}\right)\right.}\right)} d \xi_{1} d \xi_{2} d \xi_{3}}{r^{3 / 2} N^{3 s}},
$$

where

and

$$
Q_{3}\left(\xi_{1}, \xi_{2}, \xi_{3}\right):=\frac{e^{i t\left(p\left(\xi_{1}\right)+p\left(\xi_{2}+\xi_{3}\right)-p\left(\xi_{1}+\xi_{2}+\xi_{3}\right)\right)}-1}{p\left(\xi_{1}\right)+p\left(\xi_{2}+\xi_{3}\right)-p\left(\xi_{1}+\xi_{2}+\xi_{3}\right)}
$$

$$
\begin{aligned}
\left\|G_{2}\right\|_{H^{s}} & \lesssim \frac{\left\|\iiint_{\max _{j=1,2,3}\left|\xi_{j} \pm N\right|<r} \frac{e^{i x\left(\xi_{1}+\xi_{2}+\xi_{3}\right)}}{\left|\xi_{2}+\xi_{3}\right|+N^{-2}} d \xi_{1} d \xi_{2} d \xi_{3}\right\|_{L_{x}^{2}(\mathbb{R})}}{r^{3 / 2} N^{2 s+3}} \\
& \lesssim r^{-1} N^{-2 s-3} \iint_{\max _{j=2,3}\left|\xi_{j} \pm N\right|<r}\left(\left|\xi_{2}+\xi_{3}\right|+N^{-2}\right)^{-1} d \xi_{2} d \xi_{3} \\
& \lesssim N^{-2 s-3} \log N .
\end{aligned}
$$

Consequently, we get

$$
\frac{N^{-2 s-3 / 2}}{\log N}\left(1-\left(\frac{\log N}{N^{3 / 4}}\right)^{2}\right) \lesssim\left\|G_{1}\right\|_{H^{s}}-\left\|G_{2}\right\|_{H^{s}} \lesssim\left\|A_{3}(f)\right\|_{H^{s}} \lesssim 1
$$

whence deriving $s \geq-3 / 4$ (via letting $N \rightarrow \infty$ ) - a contradiction to $s<-3 / 4$. This completes the proof of Theorem 1.2 (ii).

\section{REFERENCES}

1. J. P. Albert, J. L. Bona and J. M. Restrepo, Solitary wave solutions of the Benjamin equation, SIAM J. Appl. Math. 59 (1997), 2139-2161.

2. B. Alvarez-Samaniego and J. Angulo, Existence and stability of periodic travelling-wave solutions of the Benjamin equation, Commun. Pure Appl. Anal. 4 (2005), 367-388.

3. J. Angulo, Existence and stability of solitary wave solution of the Benjamin equation, $J$. Differentional Equations 152 (1999), 136-159.

4. I. Bejenaru and T. Tao, Sharp well-posedness and ill-posedness results for a quadratic nonlinear Schrödinger equation, J. Funct. Anal. 233 (2006), 228-259.

5. T. B. Benjamin, A new kind of solitary waves, J. Fluid Mech. 245 (1992), 401-411. 
6. J. Bourgain, Fourier retsriction phenomena for certain lattice subsets and applications to nonlinear evolution equations, I. Schrödinger equations; II. The KdV-equation, Geom. Funct. Anal. 3 (1993), 107-156; 209-262.

7. J. Bourgain, Periodic Korteweg de Vries equation with measures as initial data, Selecta Math. (N.S.) 3 (1997), 115-159.

8. N. Burq and F. Planchon, On well-posedness for the Benjamin-Ono equation, Math. Ann. 340 (2008), 497-542.

9. H. Chen and J. L. Bona, Existence and asymptotic properties of solitary-wave solutions of the Benjamin-type equations, Adv. Diff. Eqns. 3 (1998), 51-84.

10. M. Christ, J. Colliander and T. Tao, Asymptotics, frequency modulation, and low regularity ill-posedness for canonical defocusing equations, Amer. J. Math. 125 (2003), 1235-1293.

11. J. Ginibre, Y. Tsutsumi and G. Velo, On the Cauchy problem for the Zakharov system, J. Funct. Anal. 151 (1997), 384-436.

12. A. Grünrock, New applications of the Fourier restriction norm method to wellposedness problem for nonlinear evolution equations, Dissertation. 2002.

13. B. Guo and Z. Huo, The well-posedness of the Korteweg-de Vries-Benjamin-Ono equation, J. Math. Anal. Appl. 295 (2004), 444-458.

14. A. D. Ionescu and C. E. Kenig, Global well-posedness of the Benjamin-Ono equation in lowregularity spaces, J. Amer. Math. Soc. 20 (2007), 753-798.

15. C. E. Kenig, Recent progress in the well-posedness of the Benjamin-Ono equation, Rev. Un. Mat. Argentina 46 (2005), no. 2, 105-112 (2006).

16. C. E. Kenig, G. Ponce and L. Vega, The Cauchy problem for the Korteweg-de Vries equation in Sobolev spaces of negative indices, Duke Math. J. 71 (1993), 1-21.

17. C. E. Kenig, G. Ponce and L. Vega, A bilinear estimate with applications to the KdV equation, J. Amer. Math. Soc. 9 (1996), 573-603.

18. H. Kozono, T. Ogawa and H. Tanisaka, Well-posedness for the Benjamin equations, J. Korean Math. Soc. 38 (2001), 1205-1234.

19. F. Linares, $L^{2}$ global well-posedness of the initial value problem associated to the Benjamin eqaution, J. Differentional Equations 152 (1999), 377-393.

20. F. Linares and M. Scialom, On generalized Benjamin type equations, Discrete and Continuous Dynamical Systems 12 (2005), 161-174.

21. K. Nakanishi, H. Takaoka, Y. Tsutsumi, Counterexamples to bilinear estimates related with the KdV equation and the nonlinear Schrödinger equation, Methods Appl. Anal. 8 (2001), 569-578.

22. A. Petersen, J. Hyman and J. Restrepo, Nonlinear, dispersive partial differential equations, http://math.lanl.gov/SummerPrograms/Reports2004/peterson.pdf.

23. T. Tao, Multilinear weighted convolution of $L^{2}$ functions, and applications to nonlinear dispersive equations, Amer. J. Math. 123 (2001), 839-908.

24. T. Tao, Nonlinear dispersive equations: local and global analysis, CBMS Regional Conference Series in Mathematics, 106, American Mathematical Society, Providence, RI, 2006.

25. N. Tzvetkov, Remark on the local ill-posedness for KdV equation, C. R. Acad. Sci. Paris $\mathbf{3 2 9}$ (1999), 1043-1047.

Institute of Applied Physics and Computational Mathematics, P.O.Box 8009, Beijing 100088, CHINA

E-mail address: chenwg@iapcm.ac.cn

Department of Mathematics and Statistics, Memorial University of Newfoundland, St. John's, NL A1C 5S7, CANAdA

E-mail address: jxiao@math.mun.ca 